\title{
Cloud and thermodynamic parameters retrieved from satellite ultraspectral infrared measurements
}

\author{
Daniel K. Zhou* ${ }^{1}$, William L. Smith ${ }^{2,3}$, Allen M. Larar ${ }^{1}$, Xu Liu ${ }^{1}$, Jonathan P. Taylor ${ }^{4}$, Peter \\ Schlüssel $^{5}$, L. Larrabee Strow ${ }^{6}$, and Stephen A. Mango ${ }^{7}$ \\ ${ }^{1}$ NASA Langley Research Center, Hampton, VA, USA \\ ${ }^{2}$ Hampton University, Hampton, VA, USA \\ ${ }^{3}$ University of Wisconsin-Madison, Madison, WI, USA \\ ${ }^{4}$ Met Office, Exeter, Devon, UK \\ ${ }^{5}$ EUMETSAT, Darmstadt, Germany \\ ${ }^{6}$ University of Maryland Baltimore County, Baltimore, MD, USA \\ ${ }^{7}$ NPOESS Integrated Program Office, Silver Spring, MD, USA
}

\begin{abstract}
Atmospheric-thermodynamic parameters and surface properties are basic meteorological parameters for weather forecasting. A physical geophysical parameter retrieval scheme dealing with cloudy and cloud-free radiance observed with satellite ultraspectral infrared sounders has been developed and applied to the Infrared Atmospheric Sounding Interferometer (IASI) and the Atmospheric InfraRed Sounder (AIRS). The retrieved parameters presented herein are from radiance data gathered during the Joint Airborne IASI Validation Experiment (JAIVEx). JAIVEx provided intensive aircraft observations obtained from airborne Fourier Transform Spectrometer (FTS) systems, in-situ measurements, and dedicated dropsonde and radiosonde measurements for the validation of the IASI products. Here, IASI atmospheric profile retrievals are compared with those obtained from dedicated dropsondes, radiosondes, and the airborne FTS system. The IASI examples presented here demonstrate the ability to retrieve fine-scale horizontal features with high vertical resolution from satellite ultraspectral sounder radiance spectra.
\end{abstract}

Keywords: Remote sensing, ultraspectral instrument, inversion, clouds, temperature and moisture profiles.

\section{INTRODUCTION}

Atmospheric and surface thermodynamic parameters retrieved with advanced ultraspectral remote sensors aboard Earth observing satellites are critical to general atmospheric and earth sciences research, climate monitoring, and weather prediction. The Earth Observing System (EOS) Aqua satellite with the Atmospheric InfraRed Sounder (AIRS) on board was launched on 4 May 2002. Satellite ultraspectral data (such as AIRS) has been shown to be significant for atmospheric research and monitoring our Earth environment ${ }^{1}$. The Infrared Atmospheric Sounding Interferometer (IASI) $^{2}$, on the Metop-A satellite launched on 19 October 2006 is the first of the advanced ultraspectral resolution temperature, humidity, and trace gas sounding instruments to be flown on the Joint Polar System (JPS) of NPOESS and Metop operational satellites for the purpose of improved weather, climate, and air quality observation and forecasting. The retrieval algorithms for these satellite instruments and their retrieved parameters must be validated to demonstrate the capability and accuracy of both observation and data processing systems.

Fast molecular and cloud transmittance models have been used to enable the infrared radiances used under cloudy conditions with the accuracy required for sounding retrieval. The empirical orthogonal function (EOF, or eigenvector) statistical regression retrieval algorithm has been expanded to include realistic cloud parameters (e.g., cloud top height, effective particle diameter, and optical thickness) to enable atmospheric profile retrieval using cloudy as well as cloudfree observations ${ }^{3}$. Cloud parameters as well as atmospheric profiles are simultaneously retrieved from infrared (IR) spectral radiance observations with the initially developed EOF regression algorithm using the National Polar-orbiting

*daniel.k.zhou@nasa.gov, Tel: 757-864-5663 
Operational Environmental Satellite System (NPOESS) Airborne Sounder Testbed Interferometer (NAST-I). The NAST-I fast transmittance model is a combination of the Optimal Spectral Sampling (OSS) fast molecular radiative transfer model ${ }^{4}$ and the physically-based cloud radiative transfer model based on the DIScrete Ordinate Radiative Transfer (DISORT) ${ }^{5}$ calculations performed for a wide variety of cloud microphysical properties ${ }^{6}$. A one-dimensional variational (1-D Var.) multi-variable inversion solution was added to improve an iterative background state defined by an eigenvector-regression-retrieval in order to account for nonlinearity in the 1-D variational solution ${ }^{7}$. It is shown that temperature and moisture retrievals can be achieved below optically thin clouds. For optically thick clouds, accurate temperature and moisture profiles down to cloud-top level are obtained. For both optically thin and thick cloud situations, the cloud-top height $\left(\mathrm{H}_{\mathrm{c}}\right)$ can be retrieved with high accuracy.

Retrieval algorithm development in conjunction with its performance validation is critical for the production of useful atmospheric and surface geophysical parameters as well as for the development of data-processing algorithms for future satellite instruments, such as the Cross-track Infrared Sounder (CrIS), and for demonstrating the use of the retrievals for improving numerical weather prediction. The retrieval algorithm developed with NAST-I has been adapted with a globally representative training database for EOF regression and applied to the IASI data, as well as the AIRS data, to fulfill the need for a global coverage of atmospheric profiles. This paper presents atmospheric profile results based on the single field-of-view retrieval system applied to the IASI and AIRS spectral radiance measurements during the Joint Airborne IASI Validation Experiment (JAIVEx) ${ }^{8}$. Profile retrievals are validated through comparisons with profiles observed with dedicated dropsondes, radiosondes, and determination from the NAST-I airborne Fourier Transform Spectrometer (FTS) system. The capability of satellite ultraspectral IR remote sensors to observe fine-scale horizontal thermodynamic features with high vertical resolution is demonstrated.

\section{RETRIEVAL ALGORITHM AND PERFORMANCE ANALYSIS}

The retrieval algorithm described herein only uses IR ultraspectral data; no other data from satellite or surface-based instruments nor from numerical weather analysis/prediction models is utilized in assisting or constraining the retrieval products. The IASI and AIRS fast transmittance model used herein is a combination of the Stand-alone AIRS Radiative Transfer Algorithm (SARTA) ${ }^{9}$ and the physically-based cloud radiative transfer model based on the DISORT ${ }^{5}$ calculations performed for a wide variety of cloud microphysical properties ${ }^{6}$. The retrieval algorithm used for both IASI and AIRS is based on that first developed and demonstrated with NAST-I using an EOF regression training database which consists of 15,000 profiles obtained from the SeeBor database ${ }^{10}$. The surface emissivity spectrum used with each training profile is randomly selected from a set of laboratory measured emissivity spectra for a wide variety of surface types $^{11}$. The cloud microphysical properties are also simulated. A random number generator is used to specify cloud visible optical thickness within a pre-specified range. Parameterization of balloon and aircraft cloud microphysical databas $^{12}$ is used to specify cloud effective particle radius from the cloud optical thickness (COT). An iterative 1-D Var. multi-variable inversion using the minimum-information regularization method ${ }^{7}$ is used for obtaining the final retrieval in this system. Initially, our cloudy EOF regression retrieval was developed for application to localized and seasonallylimited aircraft data gathering campaigns. For this application, a seasonally localized training with altitude-classified cloudy regression was made to constrain non-linear atmospheric and cloud retrievals. To apply these algorithms to radiance measurements from satellite instruments, an all-season global EOF regression database is used to obtain the initial profile for the 1-D Var. physical retrieval. The physical iterative retrieval can effectively, but not fully, account for non-linearity and further improve retrieval accuracy from the initial profile produced by EOF linear regression. An altitude-classified cloudy regression is replaced by an all-altitude cloudy regression thereby reducing the number of required training set regression coefficients. However, the regression coefficients are still classified with respect to cloud-free and cloudy conditions. The algorithm and details are found elsewhere ${ }^{3,7}$. Based on experience with NAST-I, it is desirable to use as many channels as possible in the retrieval process. However, some retrieval channels are excluded based on lack of a priori knowledge of contaminating trace gases and instrument noise level. The weighting functions (i.e., Jacobins) can be calculated by an analytical scheme or a numerical perturbation method. The channels in these spectral regions can be used for temperature and water vapor profile retrieval ${ }^{7,13}$.

Retrieval accuracy analysis can be estimated by taking advantage of radiative transfer model simulation in obtaining synthetic observed radiances. The truth profile (i.e., the radiosonde observation) is known and the retrieval can be directly compared with the truth to define retrieval accuracy due to; (1) instrumental noise, and (2) retrieval error 
introduced by the so-called "ill-posed" retrieval model. The disadvantage of this approach is that forward radiative transfer model error is not included. However, detailed validation in estimating both forward and inversion model errors can be fulfilled with dedicated field campaigns. Here, atmospheric conditions are coincidently captured by multiinstruments from both ground and aircraft ${ }^{14,15}$ validation sites, where dedicated coincident radiosondes were launched under satellite overpasses for a reasonable period of time ${ }^{16}$. This atmospheric profile retrieval is a solution of an "illposed" problem and its retrieval accuracy is measurement or scene dependent. Thus, it is important to estimate retrieval accuracy through globally synthetic simulations, intensive field campaigns, and validations. The retrieval accuracy analysis with a single field-of-view (FOV) retrieval algorithm reported here has been performed through synthetical simulations with IASI instrumental noise over a global dataset for both cloud-free and cloudy conditions. A testing dataset is used to simulate IASI observations for physical retrieval performance analysis. It is noted that this kind of error analysis is dependent on the testing dataset; however, the analysis can be done over a large set of samples to minimize the bias of the test dataset. Since the absolute retrieval error is observation or scene dependent, retrieval accuracy is represented by the mean difference (i.e., bias) and the standard deviation of the error (i.e., STDE) over a global dataset in three separate categories: (1) clear-sky over water, (2) clear-sky over land, and (3) cloudy conditions. Key thermodynamic parameters, such as atmospheric temperature profile, moisture profile, surface skin temperature and emissivity (for cloud-free cases) or cloud microphysical parameters (for cloudy cases), are used to indicate retrieval accuracy over a global dataset.

For cloud-free conditions, Figure 1 plots statistical results for temperature and moisture profiles retrieved from sample locations indicated in Figure 1d where the blue and red dots denote retrievals over water and over land, respectively. It is worth mentioning that this analysis is for an extremely diverse dataset including the regions (i.e., polar region, high terrain/mountains, and barren region) where retrievals normally have difficulties reaching the desired accuracy as can be obtained for some other regions (e.g., tropical oceanic regions). Retrieved profile accuracy shown in Figures 1a-1c is what we expect from this retrieval algorithm for a global coverage. It is shown that a relatively larger retrieval uncertainty in the boundary layer for both temperature and moisture profilers is present which is due to (1) lower retrieval sensitivity in the boundary layer, dependent on surface skin temperature contrast with the surface air temperature, (2) retrieved surface parameter (skin temperature and emissivity) uncertainties, and (3) the complexity of boundary layer structure and variation. The STDE and bias of surface skin temperature is listed in Table 1.

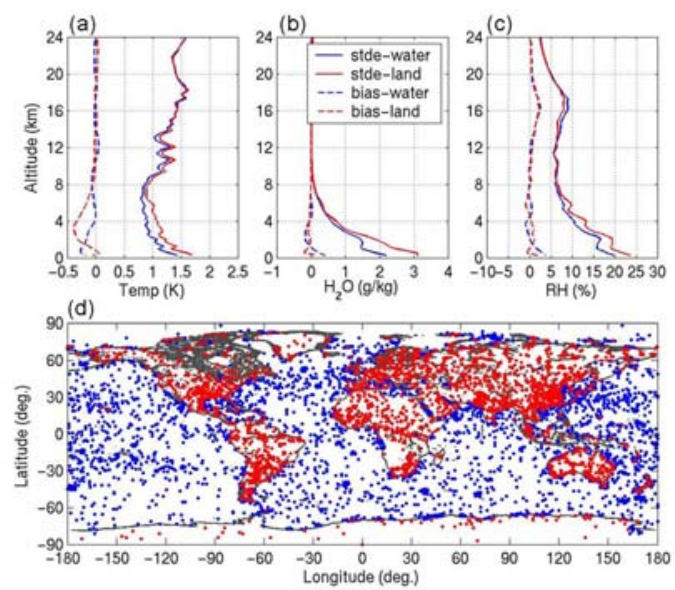

Figure 1. Retrieval error analysis over IASI simulated clear-sky observations. Panels (a)-(c) are the bias and STDE of temperature and moisture profiles for observations over water (blue curves) and land (red curves); and associated geophysical locations of the observations are indicated in panel (d) over water (blue dots) and land (red dots).

Under cloudy conditions, Figure 2 plots statistical results for temperature and moisture profiles retrieved from sample locations indicated in Figure 2d. Retrieval accuracy is shown in Figures 2a-2c. The associated cloud parameters (i.e., $\mathrm{H}_{\mathrm{c}}$, COT, and $\mathrm{D}_{\mathrm{e}}$ ) are plotted in Figure 3 and overall STDE and bias of these parameters are given in Table 1. In general, atmospheric and surface parameters are retrieved with an expected accuracy for both clear and cloudy conditions, in comparison with the "truth." The diversity of the test dataset, as represented by its standard deviation (STD), is compared with that of the retrievals from the radiances simulated for test dataset. Figure 4 shows plots of the STD of the 
test dataset (listed in Table 1) and its associated retrievals, indicating a large diversity of atmospheric conditions in this global coverage test dataset. As shown, variability of the atmospheric profiles is captured by the retrievals; however, the retrieval accuracy is better under cloud-free conditions than under cloudy conditions, especially with the moisture.
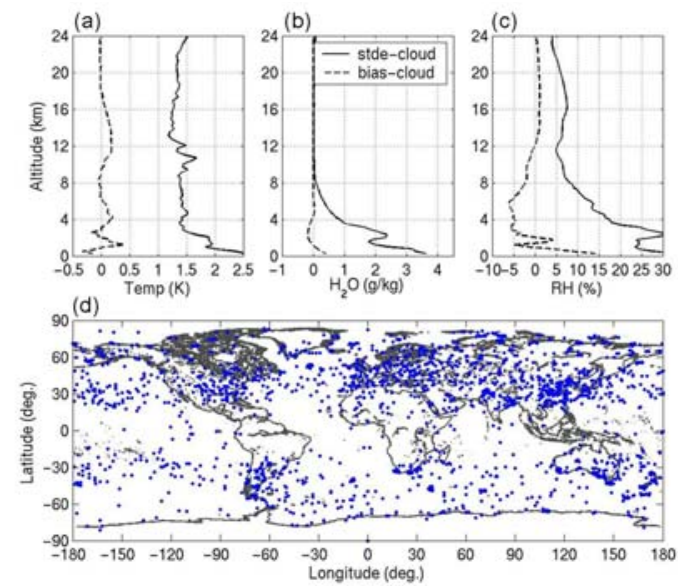

Figure 2. Retrieval error analysis over IASI simulated cloudy observations. Panels (a)-(c) are the bias and STDE of temperature and moisture profiles and associated geophysical locations of the observations are indicated in panel (d).
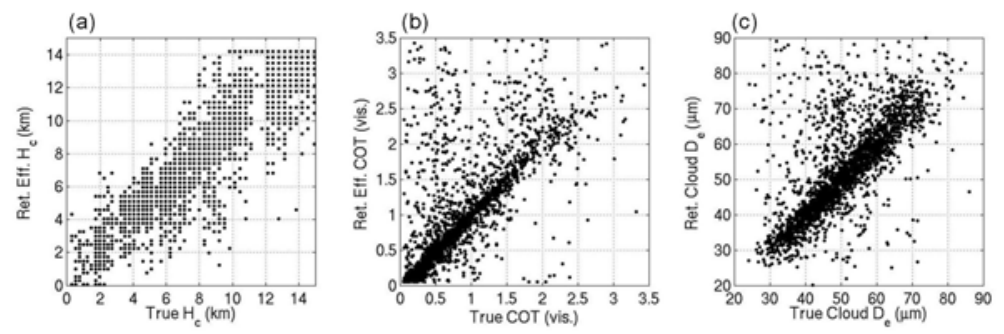

Figure 3. Retrieved cloud parameter plotted against the truth: $H_{c}, C O T$, and $D_{e}$ are in panels (a)-(c), respectively

Table 1. Surface temperature and cloud parameters retrieval error statistics

\begin{tabular}{cccc}
\hline \hline & $\begin{array}{c}\text { Clear over } \\
\text { water }\end{array}$ & $\begin{array}{c}\text { Clear over } \\
\text { land }\end{array}$ & Cloudy \\
\hline No. of Samples & 5210 & 5300 & 2337 \\
$\mathrm{~T}_{\mathrm{s}}$ Bias, $\mathrm{K}$ & 0.14 & 0.58 & -- \\
$\mathrm{T}_{\mathrm{s}}$ STDE, $\mathrm{K}$ & 0.57 & 1.51 & -- \\
$\mathrm{H}_{\mathrm{c}}$ Bias, $\mathrm{km}$ & -- & -- & 0.29 \\
$\mathrm{H}_{\mathrm{c}}$ STDE, $\mathrm{km}$ & -- & -- & 1.67 \\
COT Bias & -- & -- & 0.21 \\
COT STDE & -- & -- & 0.73 \\
$\mathrm{D}_{\mathrm{e}}$ Bias, $\mu \mathrm{m}$ & -- & -- & -1.98 \\
$\mathrm{D}_{\mathrm{e}}$ STDE, $\mu \mathrm{m}$ & -- & -- & 11.21 \\
\hline \hline
\end{tabular}

\section{JAIVEX RETRIEVAL AND INTER-COMPARISONS}

The Houston-based JAIVEx, which took place from 14 April to 4 May 2007, has gathered a wealth of useful datasets for validation of radiance observations and meteorological products from the IASI. Two aircraft, the NASA WB-57 and the Airborne Atmospheric Measurements (FAAM) BAe-146 ${ }^{15}$, operated together for several Metop-A under-flights. Flights 
were made over the Gulf of Mexico and over the US Department of Energy Oklahoma ARM-CART (Atmospheric Radiation Measurement - Cloud And Radiation Test-bed) site. Radiosondes were launched from the ARM-CART site during Metop-A overpasses. During the JAIVEx, the NASA WB-57 aircraft, together with the UK Facility for FAAM BAe-146 aircraft, made flights dedicated to IASI validation. Ultraspectral instruments, such as the NAST-I and the SHIS, flew on the NASA WB-57 aircraft to provide measurements for IASI radiance and retrieval inter-comparison. Numerous in-situ sensors and remote sensing instruments flew on the BAe-146 aircraft, and numerous dropsondes were released from the BAe-146 during under flights of IASI. Most JAIVEx data has been processed and geophysical parameters retrieved from IASI, AIRS, and NAST-I can be used to assess both retrieval accuracy and instrument performance. However, only the results from 29 April 2007 are presented here to illustrate the IASI radiometric performance and retrieval obtained during the JAIVEx. Both the WB-57 and BAe-146 aircraft flew for a period of time long enough to under fly the Metop-A and the Aqua satellites. Figures $5 \mathrm{a}$ and $5 \mathrm{~b}$ are GOES-12 infrared and visible images taken at 15:33 UTC. The IASI overpass was around 15:48 UTC with a granule of data being obtained within the yellow box and the AIRS overpass was around 19:30 UTC with a granule of data being collected within the green box. The NASA WB-57 (track with magenta line) and the FAAM BAe-146 aircraft were flown over the same location from 15:30 to 19:20 UTC. Comparisons are presented here for atmospheric soundings obtained from satellite ultraspectral instrumental measurements, airborne ultraspectral FTS measurements, and dedicated dropsondes and radiosonde observations.
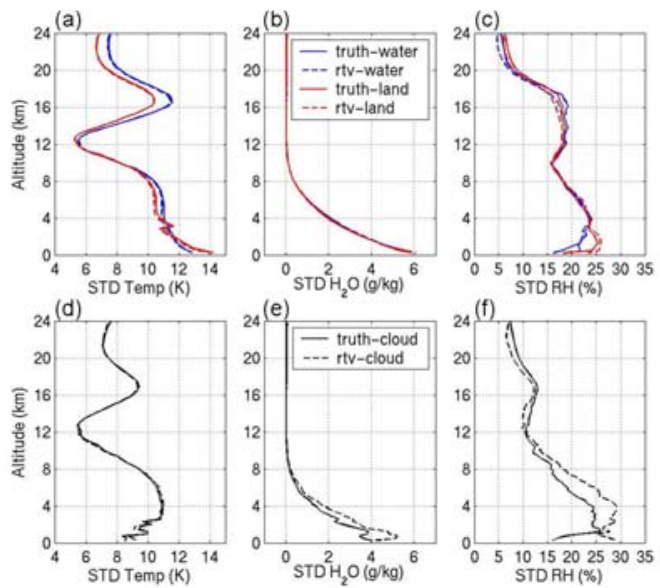

Figure 4. Standard deviation of atmospheric temperature and moisture profiles of the retrieval testing dataset. Cloud-free atmospheric conditions are plotted in panels (a)-(c); profiles over water and land are plotted in red and blue, respectively. Cloudy atmospheric conditions are plotted in panels (d)-(f).
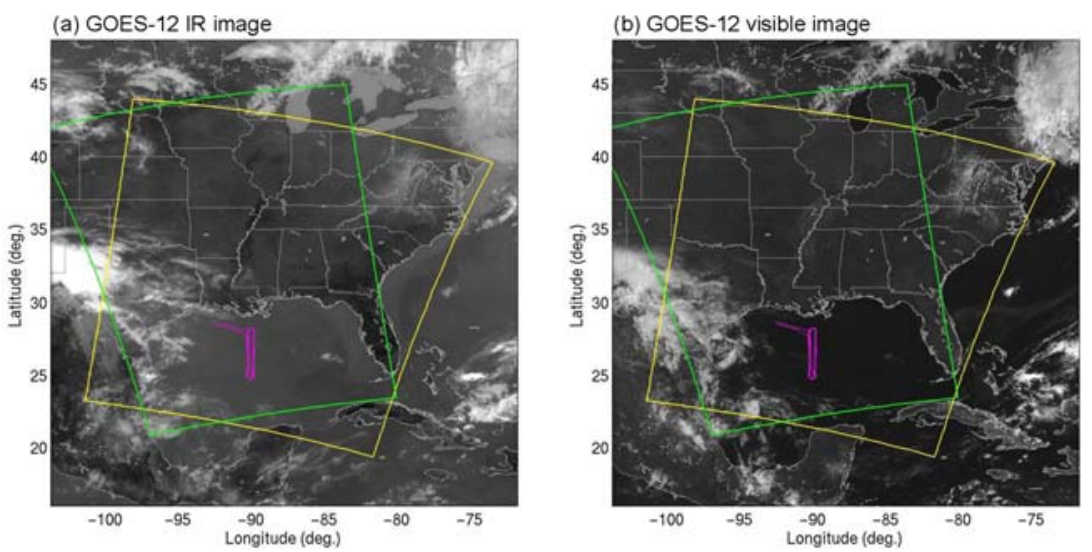

Figure 5. GOES-12 IR and visible images plotted in panels (a) and (b) with BW-57 aircraft flight track (in magenta), IASI granule (in yellow), and AIRS granule (green) boundaries 
These retrievals have gone through quality control (i.e., retrieval consistency check) based on a standard deviation of the difference between the measured and retrieval simulated brightness temperature over the spectrum of physical retrieval channels, hereafter denoted as f, spectral radiance fitting residual. This retrieval fitting residual is strongly dependent on the accuracy of retrieval parameters. Figures 6a-6d show plots of the fitting residuals, and their distributions, in the categories of clear and cloudy conditions identified by the retrieval. It is noted that they are limited to fitting residuals of less than $1.2 \mathrm{~K}$. There are 131 out of 5400 profiles in this granule that have their fitting residuals greater than $1.2 \mathrm{~K}$; most of them are due to low quality observed radiances and some complexity of cloudy conditions which are just too hard to handle with this retrieval system. Nevertheless, the percentage with a fitting residual greater than $1.2 \mathrm{~K}$ is very small, i.e., less than 3\% for this case. Fitting residuals shown in Figures 6a and $6 \mathrm{~b}$ for clear conditions are much smaller than fitting residuals shown in Figures 6c and 6d for cloudy conditions; this is expected since there are more variables to be retrieved under cloudy conditions. Radiance fitting residual samples from both clear and cloudy cases are shown in Figures 6e and 6f, respectively; and statistical analyses producing a bias and a STDE over 4786 clear and 483 cloudy samples are plotted in Figures $6 \mathrm{~g}$ and $6 \mathrm{~h}$. It is noted that the fitting residual threshold of $1.2 \mathrm{~K}$ used for this case is speculative such that there may be a few retrievals with suboptimal accuracy, especially if cloud misdetection occurs. However, this threshold can be modified by the user to filter erroneous data or more simply used as a retrieval quality indicator.
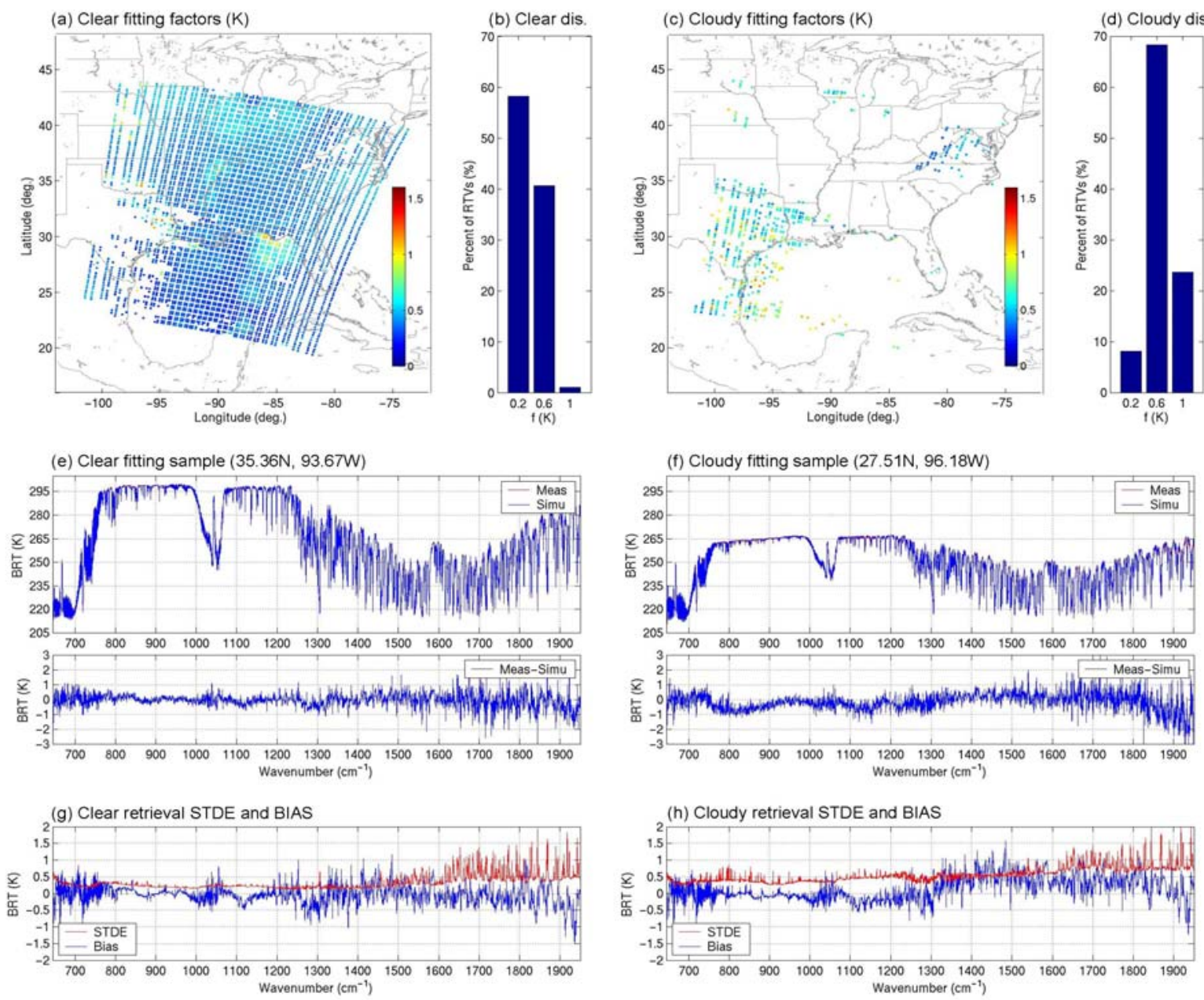

Figure 6. Retrieval fitting residuals and their distribution under clear conditions are in panels (a) and (b) while cloudy conditions are in panels (c) and (d); samples of spectral fitting of IASI bands 1 and 2 are in panels (e) and (f) for clear and cloudy conditions respectively; statistics analysis with a bias and a STDE are in panels (g) and (h) for clear and cloudy conditions respectively (see text). 
IASI retrievals include effective cloud microphysical properties, surface parameters (under clear conditions), and atmospheric temperature and moisture profiles. Cloud microphysical parameters retrieved from the IR ultraspectral data are "effective" in the sense that they depend on instrument characteristics (e.g., FOV size) and spectral dependence of the infrared sensitivity of the cloud property. For instance, observed IR radiance has a limited sensitivity variation of cloud optical depth for large values, i.e., a retrieved "effective" optical depth of an opaque can be quite different from the real optical depth of the cloud, although the cloud retrieval algorithm has been evaluated with aircraft data (e.g., dropsondes and Cloud Physics Lidar) from the THORPEX Atlantic Regional Campaign (ATReC) ${ }^{7}$, more validations for diverse cloud conditions are desired to give definitive conclusions regarding the retrieval accuracy. Unfortunately, cloud parameters derived from the JAIVEx radiance spectra can not be validated because cloud truth data is unavailable. However, IASI retrieved cloud distribution and effective cloud heights (Figure 7a) can be graphically validated using GOES-12 images (i.e., Figures 5a and 5b). Retrieved effective cloud optical depth and particle size (in diameter) are plotted in Figures 7b and 7c. It is found that cloud detection with IASI is less accurate for low cloud top heights (i.e., less than $3 \mathrm{~km}$ ) over the water. This is due to the relatively poor contrast between the cloud temperature and the underlying sea surface skin temperature. Detailed investigations are currently underway to improve the retrieval accuracy for this situation.

Surface parameters include surface skin temperature and emissivities retrieved with IASI. These parameters are critical in obtaining accurate atmospheric profiles near the surface, especially over land where emissivities have a larger variation $^{17}$. Figure 8 plots surface skin temperature and samples of emissivity images. This case was made with the BAe146 aircraft flying as low as $30 \mathrm{~m}$ above the sea; the sea surface temperature (SST) and emissivity are observed with the Airborne Research Interferometer Evaluation System (ARIES) ${ }^{15,18}$. NAST-I and ARIES observations within the IASI FOV are used for the intercomparison. IASI retrieved skin temperature over the water $(27.07 \mathrm{~N}, 90.53 \mathrm{~W})$ is $297.33 \mathrm{~K}$. This is within $0.5 \mathrm{~K}$ of the NAST-I, ARIES, and nearby buoy (station 42362 at $27.80 \mathrm{~N}, 90.67 \mathrm{~W}$ ) water temperature measurements of $297.82 \mathrm{~K}, 297.77 \mathrm{~K}$, and $297.15 \mathrm{~K}$, respectively. A sample of the water emissivity spectrum retrieved from IASI radiances is plotted in Figure $8 \mathrm{~b}$ together with a laboratory measured sea water emissivity spectrum. The agreement is similar to AIRS retrieval accuracy obtained during the EAQUATE ${ }^{14}$. Land surface skin temperature and emissivity has not been evaluated because of lack of ground truth data and the non-homogeneity of the land surface within the IASI FOV $(\sim 14 \mathrm{~km})$. However, it is evident that the land emissivity distributions differ from that of water. The small variation of water surface emissivity is expected due to its surface wind speed dependence. A sample of land emissivity, for a bare-soil spot, is plotted in Figure 8c along with a laboratory measurement of soil and sandstone emissivity. Surface emissivity images at 12 and $11 \mu \mathrm{m}$ are plotted in Figures 8d and 8e, respectively, to reveal the spectral dependence on underlying surface type.
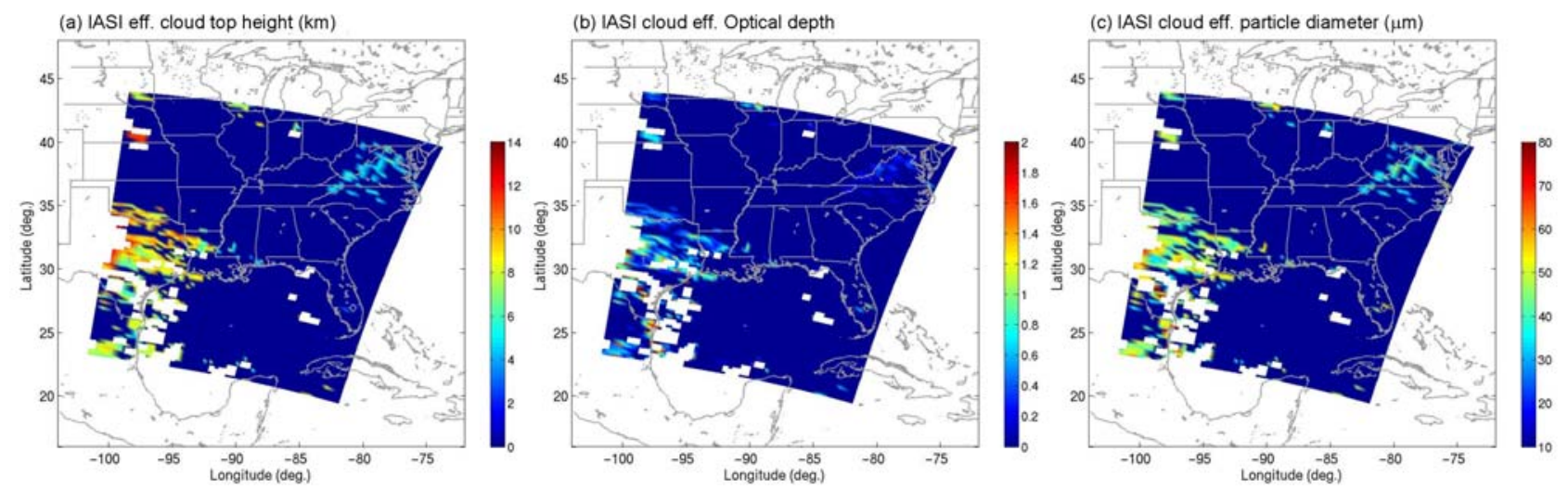

Figure 7. IASI retrieved cloud height, optical depth, and particle size are in panels (a)-(c), respectively (see text).

Atmospheric temperature and moisture distributions have been retrieved from IASI radiance measurements and validated through comparison with radiosondes, dropsondes and the retrievals from NAST-I and AIRS. A coherent distribution is shown even though the retrieval is performed independently for each individual FOV without any correlation between the neighboring FOVs. In order to obtain coincident sounding profiles for retrieval validation, the NASA WB-57 and FAAM BAe-146 aircraft flew under the Metop-A satellite to provide NAST-I radiance observations very similar to IASI and dropsonde observations. Figures 9a and 9b show cross sections of temperature deviation from their granule mean 
and relative humidity; geophysical locations of the cross section (dashed curve) are plotted in Figure 9c. One radiosonde launched at 12 UTC near the track (i.e., cross section) is also plotted in Figure 9d against a co-located IASI retrieval, despite there being a several hour gap between the IASI and radiosonde observations. Sondes were dropped from BAe146 aircraft at the Metop-A overpass time. Two of these dropsondes, one at the most southern and the other at the most northern flight locations shown on the map (Figure 9c), are plotted in Figures 9e and 9f against co-located IASI retrievals. Although these locations are not far apart, each individual comparison is made for a very different atmospheric condition. IASI retrievals captured the atmospheric variations, especially for water vapor, between these locations. The variation of water vapor from the upper atmosphere down through the boundary layer to the surface is captured as confirmed by the dropsonde and radiosonde observations. The differences between IASI retrievals and dropsonde observations are within the accuracy estimated in Section 2. Large peak-to-peak differences are due to the higher vertical resolution of dropsondes, the difference being within the retrieval accuracy estimated in Section 2 when layer averaged profiles are compared (not shown here).
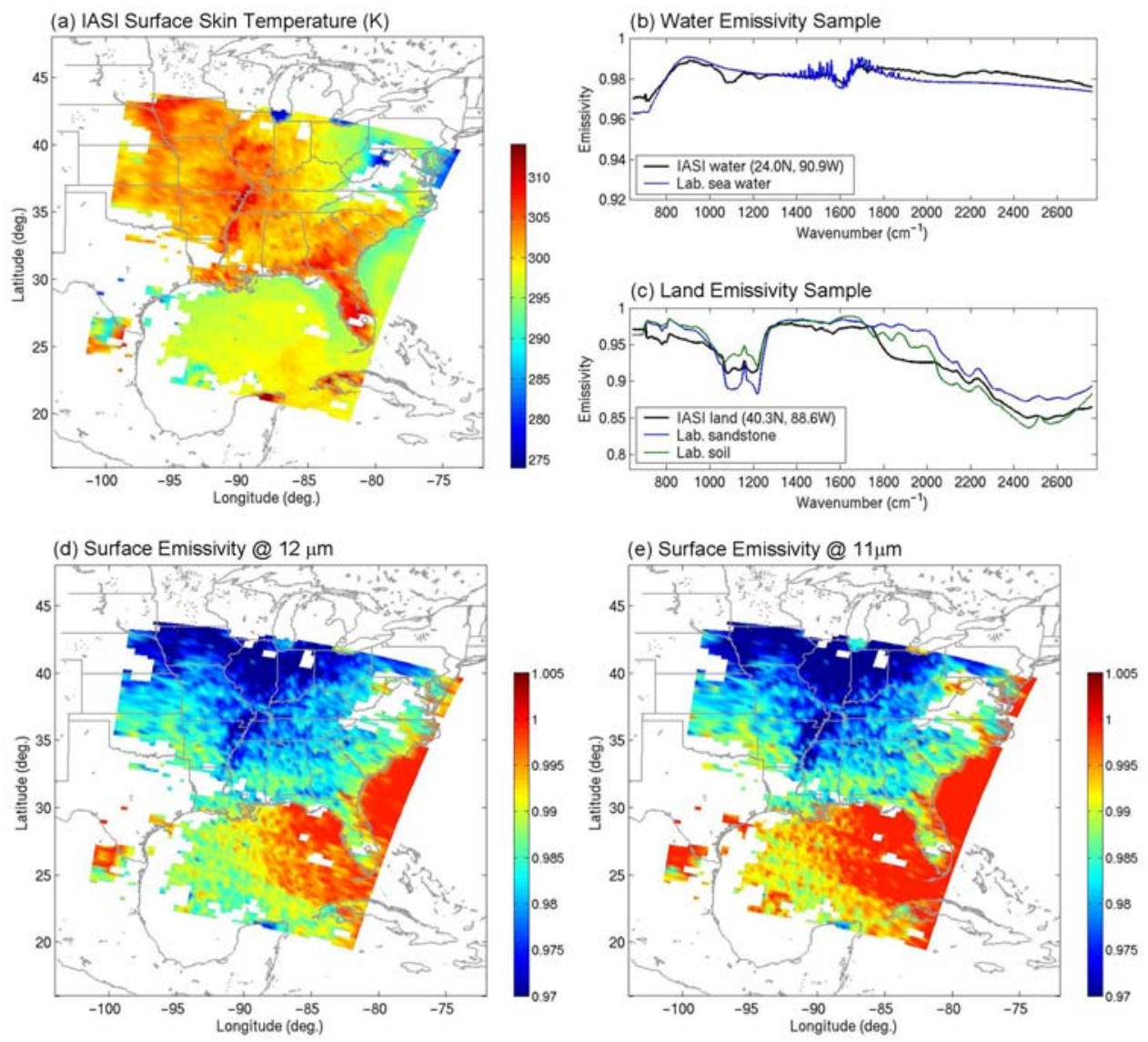

Figure 8. Surface parameters retrieved from IASI. Panel (a) is surface skin temperature, panels (b)-(c) are samples of emissivity spectra of water and land respectively, and panels (d)-(e) are emissivity distributions at $12-\mu \mathrm{m}$ and $11-\mu \mathrm{m}$, respectively. Notice that white areas were covered with clouds

Observations with AIRS on board the Aqua satellite are also used to validate the atmospheric spatial variation observed with IASI, despite their different temporal measurement time between 15:48 UTC (i.e., 10:48 am local time) for IASI and 19:30 UTC (i.e., 2:30 pm local time) for AIRS observations. AIRS retrieval is obtained with the same retrieval algorithm described in Section 2. In order to make a comparison between IASI and AIRS at the same geophysical location within a common boundary shown in Figure 5, AIRS retrievals are interpolated to IASI FOVs as shown in Figures 10a-10c, with a cross section at the same geophysical locations as that of IASI shown in Figure 9. The differences between IASI and AIRS retrievals are also plotted in Figures 10d-10f. Very similar spatial distributions of temperature and moisture cross sections are shown despite of a diurnal effect (or a temporal difference) which 
contributes to a warmer boundary temperature found in AIRS retrieval, especially over land. A warmer land surface temperature is greatly pronounced with AIRS observation while the sea surface temperature remained almost the same. Instrument measurement performance, such as spectral resolution and noise level, also contribute to the retrieval performance and difference. Nevertheless, in this particular case for the period between the IASI and AIRS observations, the field evolution is subtle while the atmospheric variation from location to location is strong as shown by both the IASI and AIRS retrievals.
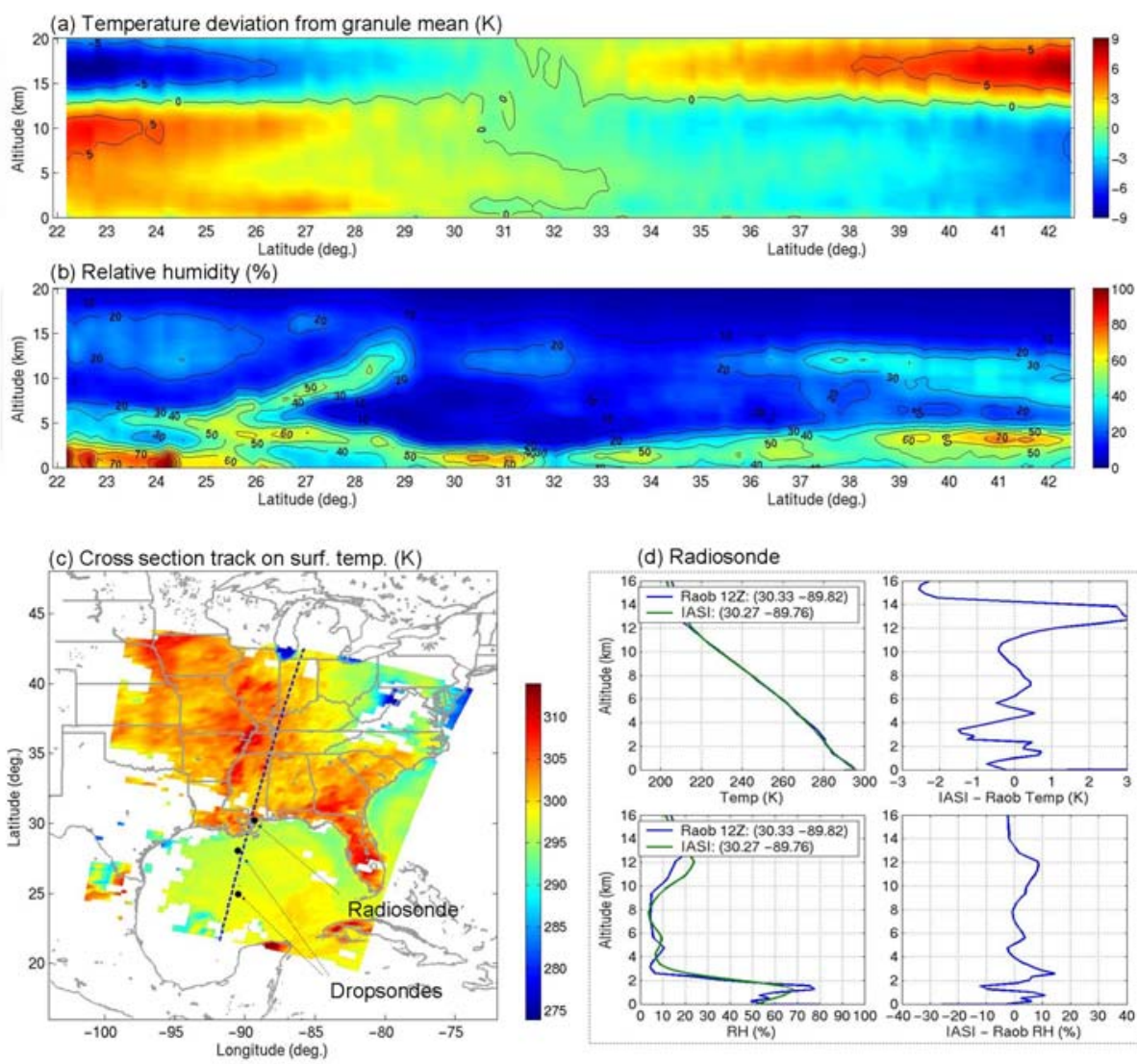

(d) Radiosonde

(e) Dropsonde \#1
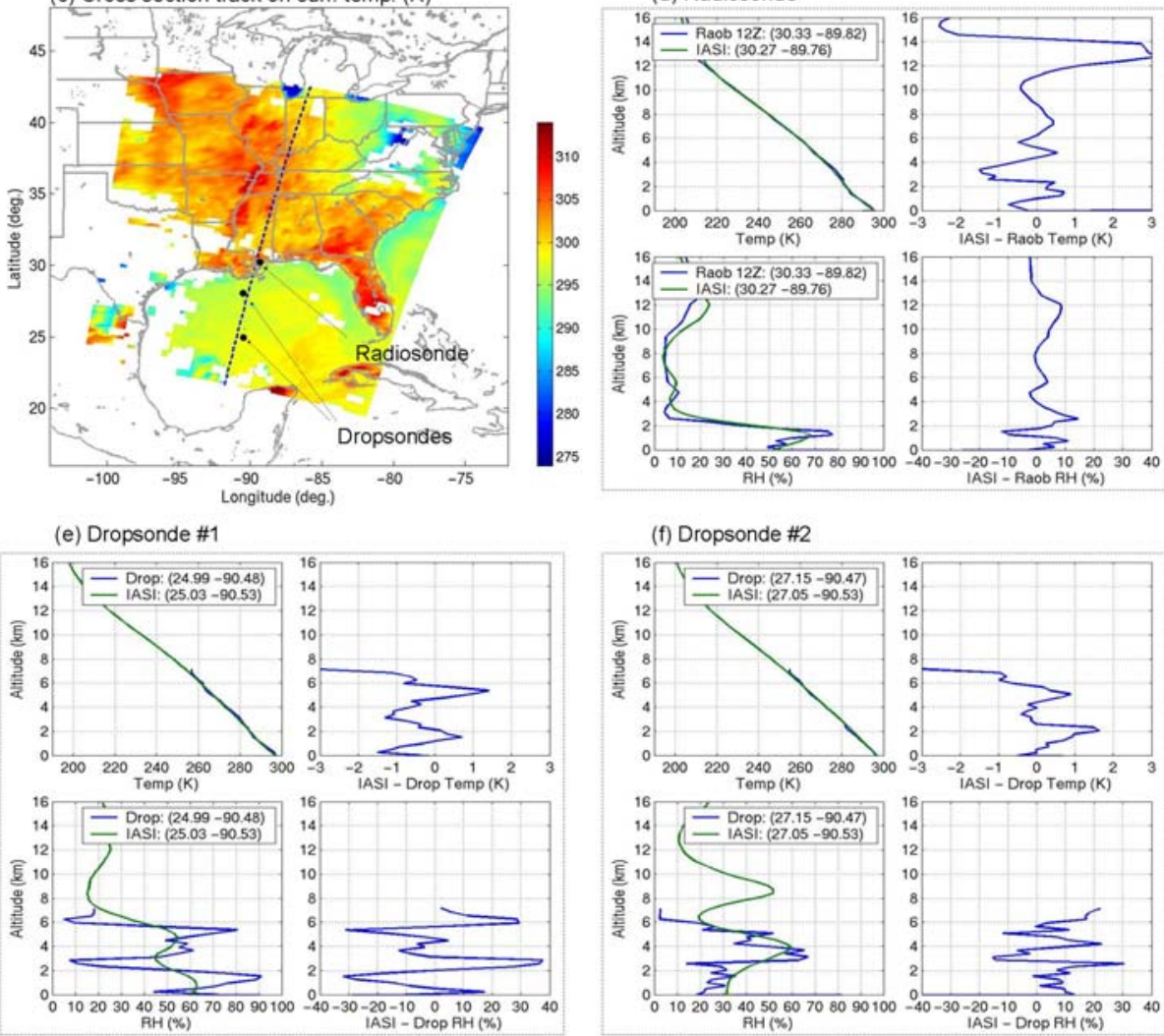

(f) Dropsonde \#2
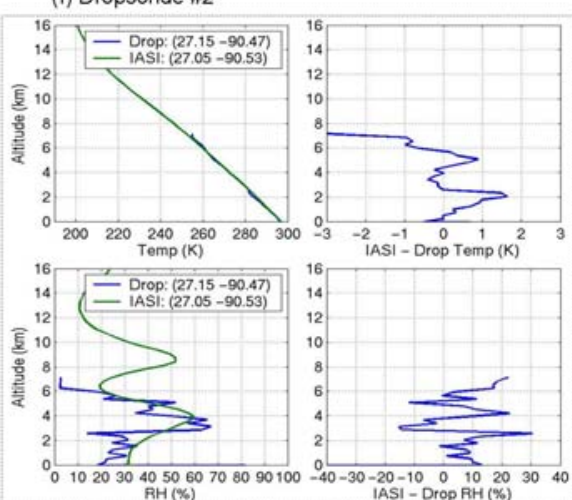

Figure 9. IASI cross sections of (a) temperature deviation from the mean and (b) relative humidity plotted from the cross section track shown in dashed curve on (c) surface skin temperature map. Locations of two dropsondes and on one radiosonde near to the track are also shown. Panels (d)-(f) plot the inter-comparisons between the sounders and IASI retrievals. 

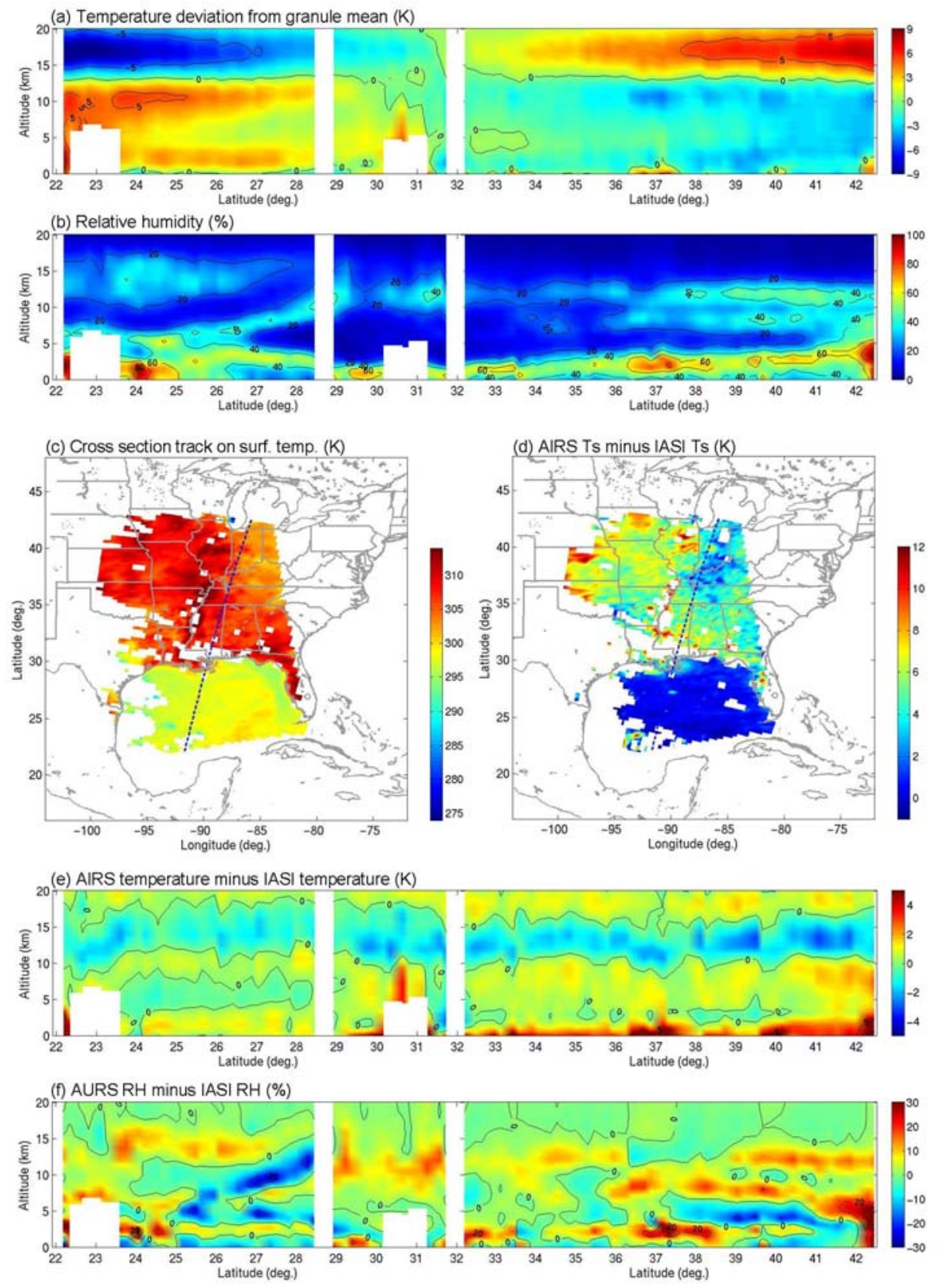

Figure 10. AIRS cross sections of (a) temperature deviation from the mean and (b) relative humidity plotted from the cross section track shown in dashed curve on (c) AIRS surface skin temperature map. The difference between AIRS and IASI in surface temperature (Ts), temperature, and relative humidity (RH) are found in panels (d)-(f) respectively.

The WB-57 aircraft IASI under-flights within the cross sections shown in Figures 9 and 10 are used to verify the subtle field evolution which occurred between the time of the IASI and AIRS observations. NAST-I relative humidity crosssections are plotted in Figure 11 as a function of time. The RH difference between AIRS and IASI is plotted in Figure 12a and compared with that observed by NAST-I plotted Figure 12b (i.e., the difference between Leg 1 and Leg 8). Their difference is mainly due to (1) instrumental differences between IASI (Figure 11a) and AIRS (Figure 11c), (2) spatial resolution differences between NAST-I and IASI (and/or AIRS), and (3) retrieval uncertainty including radiative transfer model differences (i.e., SARTA for AIRS and IASI while OSS for NAST-I). Nevertheless, these differences are within the goal of RH retrieval accuracy. The spatial characteristics of the field are very similar between NAST-I, IASI, and AIRS. The temporal variation between IASI and AIRS observation times is revealed by NAST-I measurements. 


\section{SUMMARY AND FUTURE WORK}

The international experiment, JAIVEx, was successfully conducted for validating ultraspectral satellite measurements. Experimental data were collected during the campaign; these data are used for IASI and AIRS geophysical product validation and for intercomparison of these products accounting for time discrepancies. A state-of-the-art retrieval algorithm, dealing with both clear and cloudy conditions, has been developed and applied to IASI and AIRS radiance measurements. Initial validation using JAIVEx data indicates that fine vertical structure of atmospheric field is retrieved with satellite IR ultraspectral remote sensors. Surface, cloud, and atmospheric structure and variation are captured in IASI retrievals. AIRS retrievals are interpolated to the IASI horizontal resolution for inter-comparison at the same geophysical locations; a temporal variation between AIRS and IASI observations is considered and verified with JAIVEx aircraft observations to account for atmospheric variation within the temporal gap between two satellites. First results show that both IASI and AIRS have a similar vertical resolving power with atmospheric spatial structure being captured by both instruments. In summary, JAIVEx has provided a first-hand validation dataset, which is being used for a rapid evaluation of IASI measurement capability and the further development of its data processing systems. Additional validation analyses are ongoing to provide more-definitive conclusions

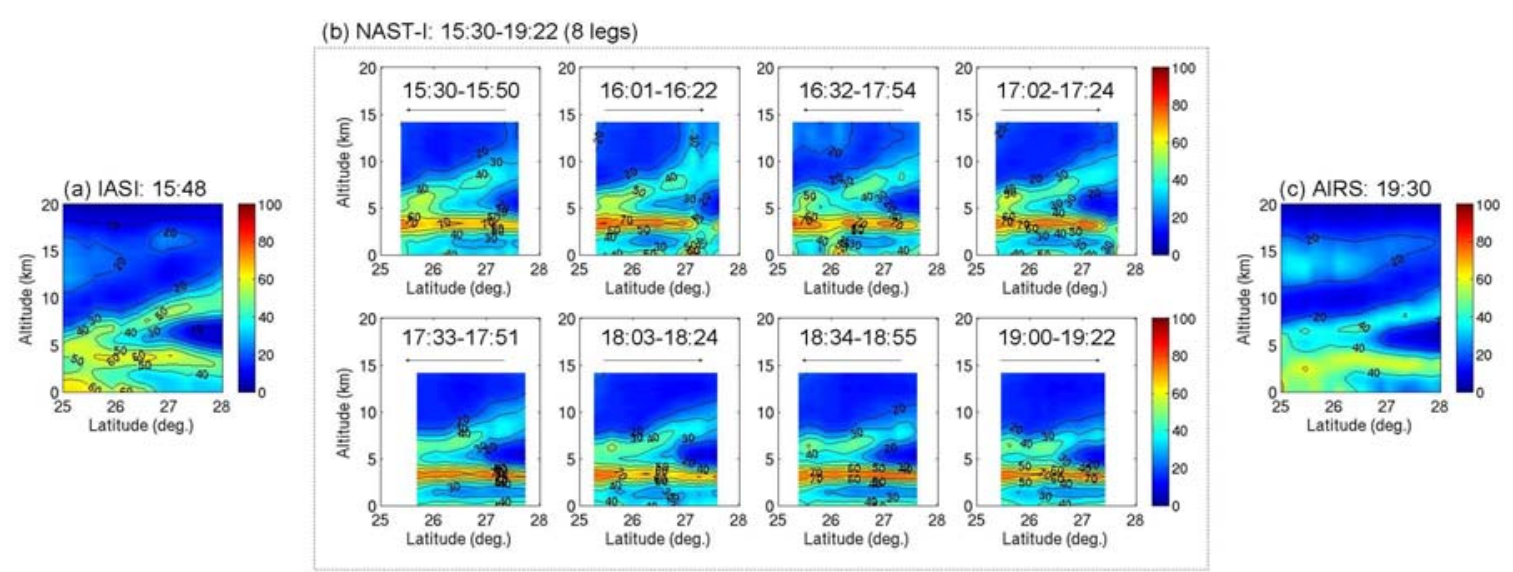

Figure 11. (a) IASI RH cross-section near aircraft flight domain, (b) NAST-I RH shown as a function of time: aircraft flight direction of each leg is shown by the arrow and the flight leg duration (in UTC) is labeled, and (c) AIRS RH cross-section interpolated to IASI FOV locations.
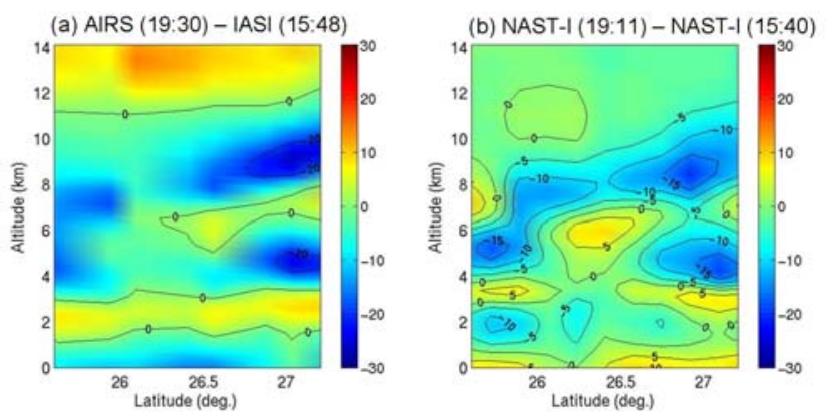

Figure 12. RH evolution characteristic between AIRS and IASI measurements observed by (a) AIRS at 19:30 UTC and IASI at 15:48 UTC, and (b) by NAST-I at 19:11 UTC and 15:40 UTC.

\section{ACKNOWLEDGEMENTS}

The authors greatly appreciate the contributions of the NASA Langley Research Center, the Space Science and Engineering Center of the University of Wisconsin - Madison, and the UK Met Office. The NAST-I program is supported by the Integrated Program Office, NASA Headquarters, and NASA Langley Research Center. The FAAM is 
jointly funded by the UK Met Office and the Natural Environment Research Council. IASI has been developed and built under the responsibility of the Centre National d'Etudes Spatiales (CNES). It is flown onboard the Metop satellites as

part of the EUMETSAT Polar System. The IASI L1 data are received through the EUMETCast near real time data distribution service.

\section{REFERENCES}

1. C. Chahine, and Coauthors, "AIRS: improving weather forecasting and providing new insights into climate", Bulletin of the American Meteorological Society, 87, 911-926 (2006).

2. D. Blumstein, G. Chalon, T. Carlier, C. Buil, P. Hebert, T. Maciaszek, G. Ponce, and T. Phulpin, "IASI instrument: technical overview and measured performances”, SPIE Proc. 5543, 196-207 (2004).

3. D. K. Zhou, W. L. Smith, X. Liu, A. M. Larar, H.-L. Huang, J. Li, M. J. McGill, and S. A. Mango, “Thermodynamic and cloud parameters retrieval using infrared spectral data”, Geophys. Res. Lett., 32, L15805, doi:10.1029/2005GL023211 (2005).

4. J. L. Moncet, and Coauthors, "Algorithm theoretical basis document (ATBD) for the Cross Track Infrared Sounder (CrIS) environmental data records (EDR)”, V1.2.3, AER Document Number P882-TR-E-1.2.3-ATBD-03-01 (2003).

5. K. Stamnes, S.-C. Tsay, W. Wiscombe, and K. Jayaweera, "Numerically stable algorithm for discrete-ordinatemethod radiative transfer in multiple scattering and emitting media”, App. Opt., 27, 2502-2509 (1988).

6. P. Yang, B. C. Gao, B. A. Baum, Y. Hu, W. J. Wiscombe, S.-C. Tsay, D. M. Winker, and S. L. Nasiri, "Radiative Properties of cirrus clouds in the infrared $(8-13 \mu \mathrm{m})$ spectral region”, J. Quant. Spectros. Radiat. Transfer, 70, 473504, 2001.

7. D. K. Zhou, W. L. Smith, Sr., X. Liu, A. M. Larar, S. A. Mango, and H.-L. Huang, "Physically retrieving cloud and thermodynamic parameters from ultraspectral IR measurements”, J. Atmos. Sci., 64, 969-982 (2007).

8. W. Smith, A. Larar, J. Taylor, H. Revercomb, S. Kireev, D. Zhou, X. Liu, D. Tobin, S. Newman, P. Schluessel, A. Clough, S. Mango, and K. St. Germain, "Joint Airborne IASI Validation Experiment (JAIVEx) - an overview", Proc. Int. ATOVS Study Conf. XVI, Angra dos Reis, Brazil, CIMSS, University of Wisconsin-Madison (2008).

9. L. L. Strow, S. E. Hannon, S. De Souza-Machado, H. E. Motteler, and D. Tobin, "An overview of the AIRS radiative transfer model”, IEEE Trans. Geosci. Remote Sensing, 41, 303-313 (2003).

10. S. W. Seemann, E. E. Borbas, R. O. Knuteson, G. R. Stephenson, and H.-L. Huang, "Development of a global infrared land surface emissivity database for application to clear-sky sounding retrievals from multi-spectral satellite radiance measurements”, J. Appl. Meteorol. Climatol., 47, 108-123 (2008).

11. J. W. Salisbury, and D. M. D'Aria, "Emissivity of terrestrial material in the 8-14 $\mu \mathrm{m}$ atmospheric window", Remote Sens. Environ., 42, 83-106 (1992).

12. A. Heymsfield, J. S. Matrosov, and B. Baum, "Ice water path-optical depth relationships for cirrus and deep stratiform ice cloud layers”, J. Appl. Meteor., 42, 1369-1390 (2003).

13. D. K. Zhou, W. L. Smith, J. Li, H. B. Howell, G. W. Cantwell, A. M. Larar, R. O. Knuteson, D. C. Tobin, H. E. Revercomb, and S. A. Mango, "Thermodynamic product retrieval methodology for NAST-I and validation”, App. Opt., 41, 6957-6967 (2002).

14. D. K. Zhou, W. L. Smith, V. Cuomo, J. P. Taylor, C. D. Barnet, P. Di Girolamo, G. Pappalardo, A. M. Larar, X. Liu, S. M. Newman, C. Lee, and S. A. Mango, "Retrieval validation during the European Aqua Thermodynamic Experiment”, Q. J. R. Meteorol. Soc. 133, 203-215 (2007).

15. J. P. Taylor, and Coauthors, "EAQUATE - An international experiment for hyper-spectral atmospheric sounding validation”, Bulletin of the American Meteorological Society, 89, 203-218 (2008).

16. D. C. Tobin, H. E. Revercomb, R. O. Knuteson, B. M. Lesht, L. L. Strow, S. E. Hannon, W. F. Feltz, L. A. Moy, E. J. Fetzer, and T. S. Cress, "Atmospheric Radiation Measurement site atmospheric state best estimates for Atmospheric Infrared Sounder temperature and water retrieval validation”, J. Geophys. Res., 111, D09S14 doi:10.1029/2005JD006103 (2006).

17. D. K. Zhou, A. L. Larar, W. L. Smith, and X. Liu, "Surface emissivity effects on thermodynamic retrieval of IR spectral radiance”, Proc. SPIE 6405, 64051H (2006).

18. S. M. Newman, J. A. Smith, M. D. Glew, S. M. Rogers, and J. P. Taylor, "Temperature and salinity dependence of sea surface emissivity in the thermal infrared”, Q. J. Royal Meteorol. Soc., 131, 2539-2557 (2005). 